\title{
INFLUENCE OF MATERIAL TYPE AND PREPARATION DEPTH ON THE FRACTURE RESISTANCE OF ENDOCROWN RESTORATION
}

\author{
Abdallah Mohamed El-Hosary*, Walid Al-Zordk**, \\ Abdelreheem abdelkader ${ }^{* * *}$ and Mohamed Hamed Ghazy ${ }^{* * * * *}$
}

\begin{abstract}
Objectives: To assess the impact of intra-coronal preparation depth and restorative material on the fracture resistance of molar restored by endo-crown restoration.
\end{abstract}

Materials and Methods: Forty root canal treated maxillary first molars were prepared for endocrown restorations. Specimens were divided according to the pulpal extension depth and material type into four groups $(\mathrm{n}=10)$ : teeth prepared with $3 \mathrm{~mm}$ pulpal extension and restored by PEEK (TP); teeth prepared with $3 \mathrm{~mm}$ pulpal extension and restored by zirconia (TZ); teeth prepared with $5 \mathrm{~mm}$ pulpal extension and restored by PEEK (FP); and teeth prepared with $5 \mathrm{~mm}$ pulpal extension and restored by zirconia (FZ). After fabrication of the restorations, each endo-crown was cemented to its corresponding tooth. All specimens were thermo-cycled $\left(5,000\right.$ cycles $-5^{\circ} \mathrm{C}$ and $\left.55^{\circ} \mathrm{C}\right)$. Each specimen was loaded till failure using universal testing machine, then the mode of failure was recorded. Two-way ANOVA and Tukey's post-hoc tests were employed for data analysis $(P<.05)$.

Results: The highest fracture load was recorded with FP group $(2578.3 \pm 337 \mathrm{~N})$ and the lowest value was recorded with TZ group $(1511.42 \pm 168 \mathrm{~N})$. Two-way ANOVA test showed a significant difference among studied groups. Post hoc test showed significant differences between each pair of groups, except a non-significant difference $(P=.787)$ between TZ and FZ groups.

Conclusions: The fracture strength of molars restored with endo-crowns can enhanced by increasing the extension depth into pulp chamber. Moreover, PEEK is considered a promising material for endo-crowns.

KEYWORDS: Endodontically-treated; Failure; Monolithic; Fracture; Endo-crown

\footnotetext{
* Fixed Prosthodontics, Faculty of Dentistry Mansoura University ** Associate Professor, Fixed Prosthodontics Dept., Faculty of Dentistry, Mansoura University, Mansoura, Egypt *** Assistant Professor, Fixed Prothodontic, Faculty of Dentistry, Mansoura University **** Professor, Fixed Prosthodontics Department, Mansoura University, Mansoura, Egypt
} 


\section{INTRODUCTION}

The restoration of root canal treated teeth is considered a continuous challenge in restorative dentistry as there are noticeable biomechanical differences in comparison with its vital equivalents, representing a multifactorial dissimilarity that includes dehydration and changes in dentin collagen result from using chemical irrigants and medicaments as well as the general weakness result from the carious lesion, previous restorations, and endodontic access cavity preparation. ${ }^{1,2}$

Many different treatment modalities have been developed for restoring non-vital teeth, including post-core systems and conventional crowns which is the preferred technique of most dentists and have been reported to exhibit a higher success rate compared with directly placed restorations..$^{3-5}$

The continuous development of bonding protocols and adhesive dentistry makes a revolution in fabrication of dental restorations. Furthermore, the amount of remaining coronal tooth structure determine the type of ceramic restoration for root canal treated teeth such as inlay, onlay, endo-crown and full crown. ${ }^{6-8}$

Endo-crown is a treatment modality which represents a restoration that combines crown and core in single unit. ${ }^{9}$ Endo-crown gets its retention through the macro-retention from the pulp chamber and the micro-retention through bonding. ${ }^{10,11}$ Teeth with insufficient coronal tooth structure, limited inter-occlusal distance that are contraindicated for conventional extra-coronal crowns could be restored with the endo-crown restorations. Endocrown restorations have multiple advantages such as simple and conservative technique because it avoid root dentine removal in order to gain retention, time saving due to fewer clinical and laboratory steps, supra-gingival margins which facilitate plaque control, and reduced treatment cost when compared to post-core-crown restoration. ${ }^{12-14}$
Selection of material used for endocrown fabrication has a great impact on the success and longevity of the restorations. New microstructural modifications were performed to enhance translucency and optical properties of the zirconia materials, such as reducing of alumina content or reducing the grains size. Moreover, adding of cubic-phase zirconia and reducing the amount of impurities and structural defects can enhance the translucency and aesthetics. The grain size is the magic key that will improve the overall polycrystalline ceramics translucency. ${ }^{15}$ Companies start to produce monolithic polychromatic multilayer blocks and blanks that mimic the color gradations presents at the natural teeth, possess high strength, need limited occlusal correction and require less tooth reduction. ${ }^{16}$ The multistep, technique sensitivity, reduced strength of the veneering material, and poor bonding between the veneer layer and the core-base are all drawbacks of bilayered restorations. ${ }^{17}$

Poly-ether-ether-ketone (PEEK) is a synthetic, tooth colored, aromatic, poly-cyclic, semi-crystalline material that produced by polymerization reaction of many small molecules, known as monomers. ${ }^{18}$ Recently these materials are widely used in the medical and dental prosthesis due to their superior mechanical properties. Large molecular mass of PEEK produces unique physical properties including toughness, high elasticity, viscoelasticity, and a tendency to form amorphous and semicrystalline structures rather than crystals. ${ }^{19}$ BioHPP is a High Performance Polymer based on PEEK raw material. It was firstly introduced as a dental material for manufacturing the super structure dentures over dental implants by the Bredent German factory. Strength of BioHPP is because it contains about $20 \%$ ceramic filler. ${ }^{18,19}$ BioHPP has elasticity which is close to human bone, that helps alleviate any stress that might develop and diminishes stress shielding..$^{20}$ Moreover, BioHPP is considered a great substitute for patients that complain allergies due to the very low water solubility (less than $0.3 \mu \mathrm{g} / \mathrm{mm}^{3}$ ) and its very low reactivity to different materials. ${ }^{21}$ 
General recommendations for endocrown preparations include $2 \mathrm{~mm}$ to $3 \mathrm{~mm}$ occlusal reduction, $90^{\circ}$ circumferential butt joint of $1-1.2 \mathrm{~mm}$ is suggested, smooth internal transitions, an occlusal divergence of 8 to $10^{\circ}$, a relatively flat pulp chamber floor with sealed canal orifices and the margins should be kept supra-gingival when possible..$^{22,23}$

There are no clear guidelines regarding the extension of pulp chamber depth needed for sufficient retention and resistance form. ${ }^{24}$ El- Damanhoury et $\mathrm{a}^{25}$ concludes that a $2-\mathrm{mm}$ intracoronal extension is enough. De Kuijper et al. ${ }^{26}$ displayed that the fracture resistance of endocrown restorations did not affected by the core depth into the pulp chamber. Ghajghouj et al. ${ }^{7}$ concluded that no correlation between different intracoronal cavity depths and fracture resistance values of endocrown. On the other side, Dartora et al. (2018), ${ }^{27}$ and Pedrollo et al. (2017) ${ }^{28}$ concluded that the greater extension of endocrowns inside the pulp chamber significantly influence the fracture resistance.

Therefore, the purpose of this in vitro study was to evaluate the effect intra-coronal preparation depth ( $3 \mathrm{~mm}$ and $5 \mathrm{~mm}$ ) and restorative material (PEEK and zirconia) on the fracture resistance of maxillary molars restored by endo-crown restoration. The null hypothesis was that the intra-pulpal extension of endo-crown would affect the fracture resistance of restored molars. Also the fracture resistance of endo-crown would be affected by the material type.

\section{MATERIAL AND METHODS}

\section{Teeth Collection and Preparation:}

Forty freshly extracted sound human maxillary molars were collected. The reason for extraction was periodontal diseases and teeth were free from any carious lesion, cracks or root resorption with similar dimensions. Each tooth was ultrasonically cleaned from calculus and stored in normal saline at room temperature. This study was approved by local Research Ethics Committee (No: A05260219).
All teeth were endodontically treated with the same operator (A.E), using rotary files (Edgefile, EdgeEndo, USA) for cleaning and shaping. Cold lateral compaction technique and resin sealer (Adseal, Meta Biomed, Korea) was used for obturation. For standardization and easy handling for specimens, every tooth was embedded in transparent epoxy resin (Chemapoxy, CMB, Egypt) $2 \mathrm{~mm}$ below CEJ.

\section{Specimen grouping:}

Teeth were divided randomly according to the pulpal extension depth and material type into four groups $(n=10)$ : teeth prepared with $3 \mathrm{~mm}$ pulpal extension and restored by PEEK (TP); teeth prepared with $3 \mathrm{~mm}$ pulpal extension and restored by zirconia (TZ); teeth prepared with $5 \mathrm{~mm}$ pulpal extension and restored by PEEK (FP); and teeth prepared with $5 \mathrm{~mm}$ pulpal extension and restored by zirconia (FZ).

\section{Teeth preparation:}

Teeth were prepared with the aid of a dental parallelometer (Marathon 103, Seayang, Korea) coupled with micromotor (Strong 204, Saeshin, Korea) and a low speed straight hand-piece (Strong AT1, Saeshin, Korea). The teeth were sectioned parallel to the occlusal surface $3 \mathrm{~mm}$ above the CEJ to provide a flat butt-joint, using a wheel diamond stone (Bluwhite, Kerr, Italy). Axial preparations involved only eliminating undercuts in the access cavity following the anatomy of the pulp chamber with a $5 \mathrm{~mm}$ intracoronal depth and approximately $4 \mathrm{~mm}$ mesio-distally and $6 \mathrm{~mm}$ bucco-palatally using flat-end taper diamond bur (BluWhite, Kerr, Italy) with an occlusal convergence of $10^{\circ}$. Occlusal table and axial wall finished and polished with the aid of finishing discs (Kit No 1.075, TorVM, Russia) to get a smooth surface preparation without any sharp line angles. A flowable composite layer (Nexcompflow, Metabiomed, Korea) was applied to the pulpal floor to seal the entrances and control the desired 3 $\mathrm{mm}$ extension of endo-crown for TP and TZ groups. The flowable composite layer was applied to each 
tooth after application and scrubbing of self-etch adhesive bond (Adper ${ }^{\mathrm{TM}}$ Single Bond 2, 3M, USA) for 30 second, and then cured for 20 second.

\section{Fabrication of endo-crown restorations:}

Each tooth was scanned (Ceramill map 600, AmannGirrbach, Germany) and the restoration was designed as monolithic restoration using digital software (Ceramill mind, AmannGirrbach, Germany) with specific features: cervico-occlusal height of $3.5 \mathrm{~mm}$ with cement gap $50 \mu \mathrm{m}$. Milling of the restoration was done using a five axis milling machine (Ceramill Motion 2 5X, Amann Girrbach, Germany). Wet milling was done for PEEK (BreCAM.BioHPP, Bredent, Germany). Dry milling was done for zirconia (KATANA UTML Kurrary, Japan) restorations. Zirconia restorations were sintered (Ceramill Therm-S, Amann Girrbach, Germany) following the manufacturer's instructions. Then, restorations were finished and glazed following the manufacturer instructions. PEEK restorations were finished and polished following the manufacturer instruction.

\section{Cementation:}

Fitting surfaces of each restoration was airabraded using alumina (Shera aluminium-oxid, Shera, Germany): $50 \mu \mathrm{m}$ at 2.5 bar for zirconia and $110 \mu \mathrm{m}, 2.5$ bar for PEEK. For zirconia restorations, a single component zirconia priming agent (Z-Primer plus, Bisco, Schaumburg, USA) was applied following manufacturer's instructions, then dried for 3-5 seconds..$^{29}$

For PEEK restorations, a conditioner (VisioLink, bredent, UK) was applied in thin layer with a brush and light cured for 90 seconds. ${ }^{30} \mathrm{~A}$ dual cure selfadhesive resin cement (Thera-cem, bisco, USA) was prepared following the manufacturer's instructions and inserted into the pulp chamber under $500 \mathrm{gm}$ constant load. Initial light polymerization to the margins for 3 seconds to aid in the removal of excess resin cement and then sample light polymerized for 20 seconds to each surface. All specimens were finished and polished.

\section{Ageing:}

Thermocycling was performed to all specimens for 5,000 cycles with a dwell time 30 seconds between $5^{\circ} \mathrm{C}$ and $55^{\circ} \mathrm{C} .{ }^{31}$

\section{Fracture resistance test:}

All specimens were individually mounted on universal testing machine (3345 machine, Instron, USA) with a load cell of $5 \mathrm{KN}$ force and a crosshead speed of $0.5 \mathrm{~mm} / \mathrm{min}$ through metallic rod with 4-mm round ball end until permanent deformation or failure of the specimen, fracture load was recorded in N. For each specimen, the failure mode was recorded and classified into; Type I (endocrown fracture); Type II (debonding of ECR), Type III (restorable remaining tooth structure, fracture above CEJ), or Type IV (non-restorable remaining tooth structure, fracture below the CEJ) ${ }^{32}$ Representative specimens were examined using scanning electron microscope (JEOL, JSM 6510 lv).

\section{Statistical analysis}

Data were analyzed using a statistical software program (IBM SPSS Statistics, v21.0; IBM Corp). The Shapiro-Wilk statistical test for normality showed normal distributions for the data. Twoway ANOVA test was used to detect the interaction between study variables $(\mathrm{P}<.001)$.

\section{RESULTS}

The highest fracture load was recorded with FP group $(2578.3 \pm 337 \mathrm{~N})$ and the lowest value was recorded with TZ group $(1511.42 \pm 168 \mathrm{~N}$ ) (Table 1). Two-way ANOVA test revealed a significant difference between studied groups (Table 2). Post hoc test showed significant differences between each pair of groups, except a non-significant difference $(P=.787)$ between $\mathrm{TZ}$ and $\mathrm{FZ}$ groups. The modes of failure classification and percentage of each group are illustrated in (Table 3). Specimens from each group were scanned by electron microscope in different magnification. Zirconia groups ( $\mathrm{T}$ and 
F) showed a high percentage of a cohesive failure (endocrown fracture), while PEEK groups ( $\mathrm{T}$ and F) showed a high percentage of an adhesive failure (endocrown debonding) as shown and described in (Figures 1 and 2)

TABLE (1): Comparison between study groups regarding the fracture resistance $(\mathrm{N})$ values.

\begin{tabular}{|c|c|c|c|c|c|}
\hline \multicolumn{2}{|r|}{$\mathrm{TP}$} & $\mathrm{TZ}$ & \multicolumn{2}{|l|}{ FP } & $\mathrm{FZ}$ \\
\hline Mean \pm SD & $2178.37 \pm 126^{\mathrm{A}, \mathrm{B}}$ & $1511.42 \pm 166^{\mathrm{B}}$ & $2578.30 \pm 337^{\mathrm{A}, \mathrm{C}}$ & \multicolumn{2}{|c|}{$1536.42 \pm 104^{\mathrm{C}}$} \\
\hline \multicolumn{6}{|c|}{$\begin{array}{l}\text { TP: PEEK endo-crown with } 3 \mathrm{~mm} \text { pupal extension; TZ: zirconia endo-crown with } 3 \mathrm{~mm} \text { pu } \\
\text { crown with } 5 \mathrm{~mm} \text { pupal extension; and zirconia endo-crown with } 5 \mathrm{~mm} \text { pupal extension }\end{array}$} \\
\hline \multicolumn{6}{|c|}{ TABLE (2): Summary of two-way ANOVA test representing the interaction between the study variables } \\
\hline Source & $\begin{array}{l}\text { Type III Sum of } \\
\text { Squares }\end{array}$ & Df & Mean Square & $\mathrm{F}$ & Sig. \\
\hline Corrected Model & $8103037.866 \mathrm{a}$ & 3 & 2701012.622 & 63.778 & .000 \\
\hline Intercept & 152276174.986 & 1 & 152276174.986 & 3595.634 & .000 \\
\hline Material (A) & 7300232.834 & 1 & 7300232.834 & 172.377 & .000 \\
\hline Depth (B) & 451392.516 & 1 & 451392.516 & 10.659 & .002 \\
\hline$A * B$ & 351412.516 & 1 & 351412.516 & 8.298 & .007 \\
\hline Error & 1524610.902 & 36 & 42350.303 & - & - \\
\hline Total & 161903823.754 & 40 & - & - & - \\
\hline Corrected Total & 9627648.768 & 39 & - & - & - \\
\hline
\end{tabular}

a. $R$ Squared $=.842($ Adjusted $R$ Squared $=.828)$

TABLE (3): The failure modes among studied groups.

\begin{tabular}{lcccc}
\hline & TP & TZ & FP & FZ \\
\hline Class I (fractured restoration) & $6(60 \%)$ & $8(80 \%)$ & $2(20 \%)$ & $2(20 \%)$ \\
\hline Class II (De-bonding) & 0 & 0 & $3(30 \%)$ & $3(30 \%)$ \\
\hline Class III ( Restorable tooth structure) & $1(10 \%)$ & $1(10 \%)$ & $3(30 \%)$ & $4(40 \%)$ \\
\hline Class IV (Non-restorable tooth structure) & $3(30 \%)$ & $1(10 \%)$ & $2(30 \%)$ & $1(10 \%)$ \\
\hline
\end{tabular}

TP: PEEK endo-crown with 3 mm pulpal extension; TZ: zirconia endo-crown with 3 mm pulpal extension; FP: PEEK endocrown with $5 \mathrm{~mm}$ pulpal extension; and zirconia endo-crown with $5 \mathrm{~mm}$ pulpal extension. 


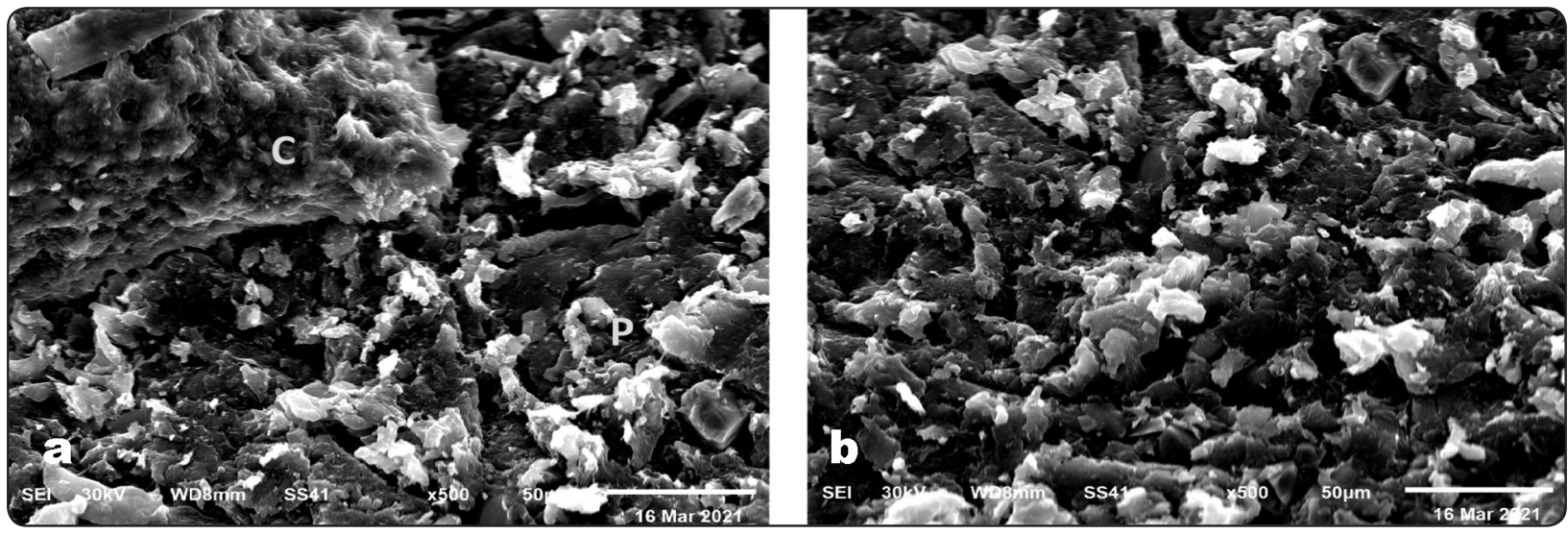

Fig. (1) Fig. (1): a) Micrograph (X 500) showing cement fragment (C) on the fitting surface of fractured PEEK (P), and b) Micrograph (X 500) the PEEK surface with no cement fragments.
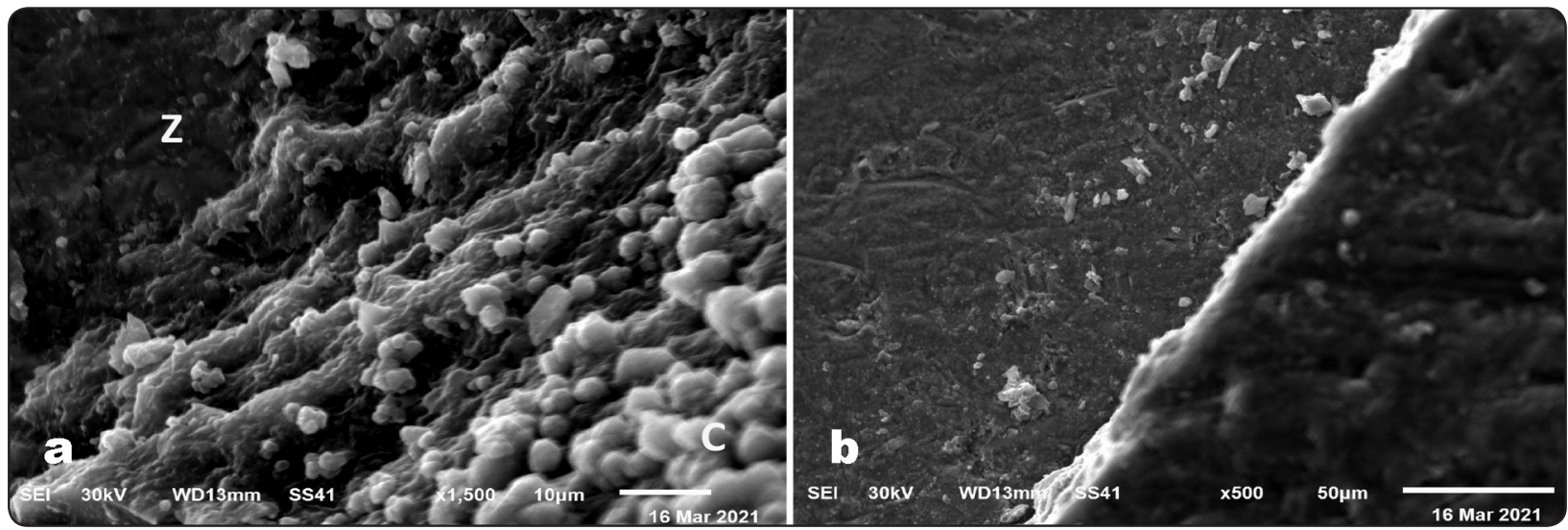

Fig. (2): a) Micrograph (X 500) showing cement fragments (c) over the zirconia surface (Z), and b) Micrograph (X500) showing the air-abraded zirconia surface.

\section{DISCUSSION}

The first null hypothesis that the intra-pulpal extension of endo-crown would affect the fracture resistance of restored molars was partially accepted because the results revealed considerable differences in the fracture resistance values between $3-\mathrm{mm}$ and 5-mm pulpal extension of PEEK endo-crowns. Also, the fracture resistance of endo-crown would be affected by the restoration type was accepted because of the significant differences between PEEK and zirconia endo-crowns.

In the current study, 5Y-PSZ (UTML katana) was selected to mimic the shade gradient determined in natural teeth due to its high content of yttria and cubic phase percentage and also for its superior strength of over $1000 \mathrm{MPa}$ and high fracture toughness of 4-5 MPa. ${ }^{29}$ BioHPP PEEK was selected for its excellent biocompatibility and superior mechanical properties as it contains about $20 \%$

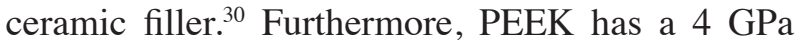
modulus of elasticity which is close to that of dentin and human bone allowing it to act as a stress breaker and absorb energy preventing it from reaching the remaining tooth structure and roots. ${ }^{33,30}$ Although monolithic PEEK features a chalky white colour that still cannot be used alone as a full-contoured aesthetic restoration owing to its low translucency and greyish pigmentation, we used it in this study to check the fracture resistance for PEEK itself and to standardize the class of the two studied material as a monolithic restorations. But overall, PEEK is 
a biocompatible material with natural tooth-coloured appearance as compared to metallic restorations. ${ }^{33}$

The results of the current study showed that teeth restored with PEEK endo-crowns had higher mean of fracture resistance than those restored with zirconia. This result may be due to properties of PEEK which has a $4 \mathrm{GPa}$ modulus of elasticity close to that of dentin and supporting human bone, making it act as a shock absorbent and stress breaker. ${ }^{33}$ Stawarczyk et $\mathrm{al},{ }^{34}$ concluded that PEEK materials showed plastic deformation without break completely due to its low Young's modules of elasticity even if compared to a high- elastic moduli material like zirconia (210 GPa). The findings of the current study are in agreement with the study of Ghajghouj et al, ${ }^{7}$ and many studies that used PEEK in different treatment modalities..$^{4,535}$ On the other hand, Elashmawy et $\mathrm{al}^{36}$ evaluated the effect of fatigue loading for four materials used as endo-crowns for mandibular molars including translucent zirconia and BioHPP PEEK and displayed that Zirconia group showed higher fracture resistance mean values than PEEK endo-crowns. This difference in results may be attributed to the use of PEEK as bilayered structure.

The results of the current study revealed a significant effect of the depth of pulpal extension into the pulp chamber on the fracture resistance of PEEK endo-crowns, while there was no significant difference for zirconia endo-crown groups. This result may be due to increase in core thickness of PEEK and a 4 GPa modulus of elasticity lead to better mechanical properties to cushion axial occlusal forces. It had been stated that restorative materials' modulus of elasticity has more effect on stress concentrations than materials' thickness. ${ }^{37}$ Ghajghouj et al, ${ }^{7}$ founded that there was no statistically significant difference between $2 \mathrm{~mm}$ and $3 \mathrm{~mm}$ core length on the fracture resistance of endo-crowns milled from PEEK, lithium silicate and zirconia reinforced lithium silicate. This result may be due to the small difference between the two core lengths. Pedrollo et al. (2017) ${ }^{28}$ evaluated the effect of different preparation depths $(2.5 \mathrm{~mm}$ and $5 \mathrm{~mm}$ ) on fracture resistance of single-rooted premolar lithium disilicate endo-crowns and concluded that the $5 \mathrm{~mm}$ deep endo-crown with a significantly higher fracture resistance than the 2.5 $\mathrm{mm}$ deep endo-crowns. Dartora et al, ${ }^{27}$ in an in vitro study and 3D finite element analysis performed to evaluate the effect of different preparation depths (5 $\mathrm{mm}, 3 \mathrm{~mm}$ and $1 \mathrm{~mm}$ ) on the fracture resistance of lithium disilicate molar endo-crowns. The highest mean value recorded among the $5 \mathrm{~mm}$ intra-coronal depth which is higher than values of $3 \mathrm{~mm}$ and 1 mm endo-crowns.

Failure modes reported in this study showed different behaviours between the study groups. This result can be explained through the microstructure and properties of each material such as modules of elasticity and bonding behaviour to tooth structure. ${ }^{38,39}$ PEEK showed only plastic deformation without complete fracture as polymer chains could distribute the plasticity under the elevated load and therefore increase the resistance to crack propagation. ${ }^{7}$

Fracture of ceramic restorations affected by the modulus of elasticity. Materials with more comparable elasticity to tooth structure have the ability to bend under load and absorb stresses more evenly, unlike rigid materials with high elastic modulus (e.g. zirconia), reproduce catastrophic failures as there are high stress concentrations at critical areas..$^{6,7,36}$

As a limitation for the current study, comparison between wide ranges of intra-pulpal preparation depths have to be studied. Also, PEEK (from esthetic point of view-still cannot be milled to full contour and requires veneering due to its chalky-white colour, low translucency and greyish pigmentation.

\section{CONCLUSIONS}

Under the limitations of this in-vitro study, the fracture strength of molars restored with endocrowns can be enhanced by increasing the extension depth into pulp chamber. Moreover, PEEK is considered a promising material for endo-crowns. 


\section{REFERENCES}

1. Soares P, Santos-Filho P, Martins L, Soares C. Influence of restorative technique on the biomechanical behavior of endodontically treated maxillary premolars, part I: fracture resistance and fracture mode. J Prosthet Dent 2008; 99:30-37.

2. Dietschi D, Duc O, Krejci I, Sadan A. Biomechanical considerations for the restoration of endodontically treated teeth: A systematic review of the literature, part ii (evaluation of fatigue behavior, interfaces, and in vivo studies). Quintessence Int 2008.

3. Cho G. Evidence-based approach for treatment planning options for the extensively damaged dentition. J Calif Dent Assoc 2004; 32: 983-990.

4. Abdullah A, Tsitrou E, Pollington S. Comparative in vitro evaluation of CAD/CAM vs conventional provisional crowns. J Appl Oral Sci 2016:24: 258-263.

5. Alhawari M, Al-zordk W, Abo-madina M. Fracture resistance of endodontically treated teeth: effect of cadcam post-core and endocrown materials. J Dent Med Sci 2019;18: 65-68.

6. Taha D, Spintzyk S, Sabet A, Wahsh M, Salah T. Assessment of marginal adaptation and fracture resistance of endocrown restorations utilizing different machinable blocks subjected to thermomechanical aging. J Esthet Restor Dent 2018;30: 319-328.

7. Ghajghouj O. Evaluation of fracture resistance and microleakage of endocrowns with different intracoronal depths and restorative materials luted with various. materials 2019;12: 25-28.

8. El-Damanhoury H, Haj-Ali R, Platt J. Fracture resistance and microleakage of endocrowns utilizing three cad-cam blocks. Oper Dent 2015;40: 201-210.

9. Dejak B, Młotkowski A. Strength comparison of anterior teeth restored with ceramic endocrowns vs custom-made post and cores. J Prosthodont Res 2018;62: 171-176.

10. Pissis P. Fabrication of a metal-free ceramic restoration utilizing the monobloc technique. Pract Periodontics Aesthet Dent 1995;7:83-94.

11. Bindl A, Mormann W. Clinical evaluation of adhesively placed Cerec endocrowns after 2 years- preliminary results. J Adhes Dent 1999; 1:255-265.

12. Naumann M, Schmitter M, Krastl . Post endodontic restoration: Endodontic post and core or no post at all? J Adhes Dent 2018;20: 19-24.
13. Robbins JW. Restoration of the endodontically treated tooth. Dent Clin North Am 2002;46:367-384.

14. Rocca G, Saratti C, Poncet A. The influence of FRCs reinforcement on marginal adaptation of CAD/CAM composite resin endocrowns after simulated fatigue loading. Odontology 2016; 104: 220-232.

15. Zhang Y, Lawn B. Evaluating dental zirconia. Dent Mater 2019;35: 15-23.

16. Kolakarnprasert, N., Kaizer, M. R., Kim, D. K. \& Zhang, Y. New multi-layered zirconias: Composition, microstructure and translucency. Dent. Mater. 35, 797-806 (2019).

17. Choi, Y.-S., Kim, S.-H., Lee, J.-B., Han, J.-S. \& Yeo, I.-S. In vitro evaluation of fracture strength of zirconia restoration veneered with various ceramic materials . J. Adv. Prosthodont. 4, 162 (2012).

18. Toth, J. M. et al. Polyetheretherketone as a biomaterial for spinal applications. Biomaterials 27, 324-334 (2006).

19. Najeeb, S., Zafar, M. S., Khurshid, Z. \& Siddiqui, F. Applications of polyetheretherketone ( PEEK ) in oral implantology and prosthodontics. J. Prosthodont. Res. 60, 12-19 (2015).

20. Bechir, E. S. et al. The advantages of BioHPP polymer as superstructure material in oral implantology. Mater. Plast. 53, 394-398 (2016).

21. Alqahtani, N. M. The Application of Polyether Ether Ketone as Post and Core : A Systematic Review. 1431-1438 (2021).

22. Hayes A, Duvall A, Roberts M. Effect of endocrown pulp chamber extension depth on molar fracture resistance. Oper Dent 2017;42:327-334.

23. Tzimas K, Tsiafitsa M, Gerasimou P, Tsitrou E. Endocrown restorations for extensively damaged posterior teeth: clinical performance of three cases. Restorative dentistry \& endodontics. 2018;43:22-38.

24. Papalexopoulos D, Samartzi T, Sarafianou A. A Thorough Analysis of the Endocrown Restoration: A Literature Review. The Journal of Contemporary Dental Practice. 2021;22:422-426.

25. ElDamanhoury H. Effect of preparation depth on the marginal and internal adaptation of computer-aided design / computer- assisted manufacture endocrowns. Oper Dent 2016; 41:607-616.

26. de Kuijper M, Cune MS, Tromp Y, et al. Cyclic loading and load to failure of lithium disilicate endocrowns: Influence 
of the restoration extension in the pulp chamber and the enamel outline. J Mech Behav Biomed Mater. 2020;105.

27. Dartora N, Ferreira D, Moris I, Brazão E, Spazin A. et al. Effect of intracoronal depth of teeth restored with endocrowns on fracture resistance: in vitro and 3-dimensional finite element analysis. J Endod 2018;44: 1179-1185.

28. Pedrollo L, Ende A, De Munck J, Suzuki T, Vieira L, et al. Biomechanical behavior of endodontically treated premolars using different preparation designs and CAD/CAM materials. J Dent 2017;59: 54-61.

29. Kolakarnprasert N, Kaizer M, Kim D, Zhang Y. New multi-layered zirconias: Composition, microstructure and translucency. Dent Mater 2019;35: 797-806.

30. Bechir E, Gioga C. The advantages of BioHPP polymer as superstructure material in oral implantology. Mater Plast 2016;53: 394-398.

31. Morresi A, D’Amario M, Capogreco M, Gatto R, Marzo $\mathrm{G}$, et al. Thermal cycling for restorative materials: Does a standardized protocol exist in laboratory testing? A literature review. J Mech Behav Biomed Mater 2014;29: 295-308.

32. Dartora N, Poole S. Mechanical behavior of endocrowns fabricated with different CAD-CAM ceramic systems. J Prosthet Dent 2021; 125:117-125.
33. Zoidis P, Bakiri E, Polyzois G. Using modified polyetheretherketone (PEEK) as an alternative material for endocrown restorations: A short-term clinical report. J Prosthet Dent 2017;117: 335-339.

34. Stawarczyk B. Polyetheretherketone - A suitable material for fixed dental prostheses? J Biomed Mater Res 2013;101: 1209-1216.

35. Haralur S. Fracture resistance of endodontically treated teeth restored with various esthetic posts. Technol Health Care 2020; 1: 1-10.

36. Elashmawy Y, Elshahawy W, Seddik M, Aboushelib $\mathrm{M}$. Influence of fatigue loading on fracture resistance of endodontically treated teeth restored with endocrowns. J Prosthodont Res 2021;65:78-85.

37. Fonseca R, Fernandes-Neto A, Correr-Sobrinho L, Soares $\mathrm{C}$. The influence of cavity preparation design on fracture strength and mode of fracture of laboratory-processed composite resin restorations. J Prosthet Dent 2007;98: 277-284.

38. Najeeb S, Zafar M, Khurshid Z, Siddiqui F. Applications of polyetheretherketone (PEEK) in oral implantology and prosthodontics. J Prosthodont Res 2015;60: 12-19.

39. Zhang Y, Lawn B. Novel zirconia materials in dentistry. J Dent Res 2018;97: 140-147. 